\title{
A Description of Earthquake Risk Perception in Intelligent Mathematics ${ }^{*}$
}

\author{
Chongfu Huang ${ }^{1,2,3}$ \\ ${ }^{l}$ Key Laboratory of Environmental Change and Natural Disaster, Ministry of Education, Beijing Normal University, \\ Beijing 100875, China \\ ${ }^{2}$ State Key Laboratory of Earth Surface Processes and Resource Ecology, Beijing Normal University, Beijing \\ 100875, China \\ ${ }^{3}$ Faculty of Geographical Science, Academy of Disaster Reduction and Emergency Management, Beijing Normal \\ University, Beijing 100875, China
}

Received June 14, 2018

Accepted June 24, 2018

\begin{abstract}
In this paper, we for the first time propose an framework of intelligent mathematics, which consists of factor space, information diffusion, and internet of intelligences. This paper demonstrates how to use intelligent mathematics to describe earthquake risk perception. The concept of earthquake risk is described in the factor space, and the knowledge of earthquake risk is formed in association learning based on information diffusion. Different knowledge systems precipitate different acquired consciousnesses. The security instinct affects the perception of risk. For a region, the risk consciousnesses of the stakeholders could be integrated with the internet of intelligences to form a consensus of the earthquake risk perception.
\end{abstract}

Keywords: earthquake risk, risk perception, intelligent mathematics, factor space, information diffusion, internet of intelligences, consciousness

\section{地震风险感知的智能数学描述方式*}

\section{黄崇福 ${ }^{1,2,3}$}

1. 北京师范大学, 环境演变与自然灾害教育部重点实验室, 北京 100875, 中国

2. 北京师范大学，地表过程与资源生态国家重点实验室，北京 100875，中国

3. 北京师范大学, 地理科学学部, 减灾与应急管理研究院, 北京 100875 , 中国

摘要: 本文首次提出了由因素空间、信息扩散和智联网组成的智能数学框架, 并演示了如何用智能数学来描 述地震风险感知。地震风险的概念在因素空间中描述，地震风险的知识在基于信息扩散的联想式学习中形成。 不同的知识体系沉淀出的后天意识明显不同。安全的本能意识影响着风险的感知。针对一个地区, 由利益相关 者的风险意识经过智联网的融合, 能形成具有一定共识性的地震风险感知。

关键词：地震风险，风险感知，智能数学，因素空间，信息扩散，智联网，意识

\section{1. 引言}

有两种地震风险感知的方式。一种是数理分析方 式, 即通过数学和物理的方式认识地震风险, 例如, 基于历史地震资料和地质及地球物理参数用概率模型 评价地震危险性, 进而认识地震风险; 另一种是主观
评价方式, 即由专家和利益相关者对所研究地区的地 震风险进行主观判断, 例如, 震害预测的层次分析 法。事实上, 地震危险性和风险承受体易损性的高度 不确定性及信息的严重不完备性, 致使数理分析中必 有大量的主观取舍, 风险感知带有分析者深深的烙 印; 另一方面, 人们以主观方式进行地震风险感知

\footnotetext{
* This project was supported by the National Natural Science Foundation of China (No. 41671502) and the National Key Research and Development Plan (2017YFC1502902).
} 
时, 不自觉地会参考大量数理分析的结果, 例如, 地 震区划图, 建筑物设防标准等等。

更一般地讲, 现代科学意义上的风险分析, 本质 上是在进行风险感知。风险分析是指, 使用有关理论 和方法, 在相关知识和数据资料的基础上, 对未来不 利事件出现的可能性、规模、影响等进行的分析 ${ }^{[1]}$ 。 用科学仪器测量风险源等获取数据时, 其实是借助机 器在感知风险世界。

因为地震预报的精度不高, 人们还无法由数据资 料、地震学理论和地震工程技术去自动感知地震风 险。既使是在风险分析模型中精细地考虑到随机不确 定性并使用模糊逻辑近似推理, 机械式感知的地震风 险也强差人意。在相当一段时间内, 地震风险感知, 仍然须人工参与。既使是使用深度学习的人工智能技 术和蚁群优化算法等复杂的数学模型, 也替代不了人 类智慧在地震风险感知中的作用, 实现不了地震风险 感知的全自动化。

要使人类制造的机器 (系统), 具人类感知能 力, 这种智能化的梦想, 与飞行的梦想一样远古。

虽然人类有了电子计算机和人工智能这类机器智 能化的有力工具, 但目前并不存在真正的机器智能。 人们谈论的智慧城市, 无非也就是自动化程度高一些

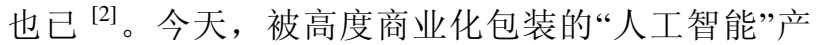
品, 其实是自动化意义下的智能, 应该被称为“灵巧” (Smart); 能创造知识的智能, 实为 “智慧” (Intelligence)。

从电子计算机问世的几十年来, 人们之所以在人 工智能的研究方面没有质的突破, 根本原因是没有一 门研究人类智能的数学。

现代数学的根基是代数学和微积分。代数学源于 量化分析静态世界的需要, 微积分源于分析动态世界 的需要。无论是代数学还是微积分, 其研究对象, 均 可在物质世界中找到原像。千千万万的代数学和微积 分的衍生数学体系, 例如集合论、概率论、拓扑学、 模糊逻辑、人工神经元网络、小波分析、支持向量 机、蚁群算法等, 都不是研究人类智能的数学。今 天, 脑科学和心理学已经很发达, 但并没有从中发展 出研究人类智能的数学, 而是只能借助现有数学做一 些简单的量化分析。

显然, 如果人类仍陷于像鸟一样飞行的尝试, 至 今也实现不了飞行的梦想。同理, 如果人类总陷于代 数学和微积分的数学体系之内, 真正的智能数学永远 不会出现, 也就无法设计出能像人一样感知地震风险 的机器。

本文在文献[2]研究的基础上, 提出智能数学的一 个框架，并用其来描述地震风险感知。

\section{2. 描述人类意识的智能数学}

智能数学, 是一个热门话题, 但常被误读。最匪 夷所思的, 莫过于将具有格结构的模糊数学称为智能 数学 ${ }^{[3]}$; 最混淆视听的, 是将发表数学、计算机和自 动化技术、人文和医学等领域文章的一本刊物取名 《Intelligent Mathematics》 ${ }^{[4]}$; 最随意的, 是称近似 分析为智能数学, 并在 Springer 出版专著《Intelligent Mathematics: Computational Analysis》 ${ }^{[5]}$ 。

以探讨智能的人工实现为已任的人工智能, 或许 是最需要智能数学支撑的学科。但人工智能的研究, 目前仍陷于代数学和微积分衍生体之中, 只不过由于 计算机科学的飞速发展, 在传统数学的基础上, 自动 化程度也能呈几何级数增长, 人们误认是人工智能取 得了突破。

把结构主义、功能主义和行为主义三大流派有机 地统一起来形成的机制主义人工智能理论 ${ }^{[6]}$, 其实是 一个以概率论、矩阵和数学映射为基础的, 处理信息 的理论体系, 仍然属于机械论的范畴。力图为该人工 智能理论提供支撑的泛逻辑学理论 ${ }^{[7]}$, 是将广义概率 论视作智能信息处理的理论依据, 扩充了现有处理不 确定性的逻辑体系，并称之为柔性信息处处理模式。

作者认为, 基于代数学和微积分的任何数学, 都 不是用来表述人类思维活动的工具, 不是智能数学。 这是因为, 人类思维活动的基础是意识, 而并非任何 意识都能在物质世界中找到原像。例如, 贞儿出生就 知道找吃的, 此时他的脑海中并无任何的概念, 驱动 其活动的, 是求生的本能意识。目前的数学, 并不提 供描述意识的工具, 也就无从谈及如何描述本能意识 和后天意义支配下的思维活动。据此, 本文给出智能 数学的定义:

定义 1. 以描述意识为基础, 以人类思维活动为研究 对象的数学, 称为智能数学。

意识(Consciousness), 通常被认为是一个心理学 名词, 主要是指人们对外界和自身的觉察与关注程 度。哲学范畴的意识是指人的头脑对于客观物质世界 的反映, 是感觉、思维等各种心理过程的总和。临床 医学中, 意识被具体化为人们对周围环境、自身状 况、周围环境事物之间的关系以及自身与环境之间相 互关系的觉察、理解与反应的能力。在机制主义的人 工智能理论中, 意识被认为是人们在一定的知识支持 下和在目标牵引下对来自外部和内部的刺激信息所产 生的觉察、理解和反应能力。

显然, 无论是考虑了生存欲等 “原意识” 的心理 学意义上的 “意识”, 还是用知识对信息进行 “觉 
察” 的人工智能关注的 “意识” , 都无法在其基础上 建立起智能数学。

心理学的 “意识” 中, 研究对象的原像仍在物质 世界中。例如, “群体欲” 这一原意识, 其原像就是 物质世界中的某种 “集合”。

人工智能关注的 “意识” , 更是离不开物质世 界，因为其依托的 “信息” 是指客体所呈现的 “状态 及其变化方式”。这里的 “客体” ，是物质世界中的 对象。

本文认为, 意识世界与物质世界, 是两个完全不 同的世界, 也不存在数学意义上的同构关系。意识世 界能部分感知物质世界, 物质世界也能部分地感知意 识世界, 但意识世界中的大多数对象, 在物质世界中 并没有原像。例如, 生存的本能意识, 并非是物质世 界中某种生灭过程的像, 因为两者完全不在一个层次 上。

在人类的思想交流中, 人们往往借助物质世界作 为媒介, 通过比喻和列举等方式, 向他人表达自己的 意识。然而, 并非任何意识, 均能通过此媒介表达出 来。很多意识性的内容, 只可意会, 不可言传。

意识世界, 并非唯心主义的产物, 而是一种客观 存在, 只不过不能完全用物质世界的观点来认识罢 了。

意识, 是人类了解宇宙和自己的最后堡垒, 也是 人类寻求突破遗传制约和优化自身物种的核心和希望 所在。显然, 无意识的机器, 不能觉醒意识的机器, 再高水平的智能, 也是一个自动化的机器。

意识, 作为非物质世界中的对象, 人类对其奥秘 知之甚少。任何试图给其下一个普适性定义的企图, 都是徒劳的。

\section{3. 智能数学基本元素}

在传统数学的版图中, 数字、算子和结构, 是基 本元素。随着数学的发展, 数字升华为用字母代表的 变量, 算子发展为复杂的数学物理模型, 实数空间结 构拓扑到一般意义的集合上, 拓扑上还可建立超拓 扑。

或许从 “概念” “知识” 和 “意识” 这三个基元 素出发, 能一步一步地生长出智能数学的版图。

\section{1. “概念” 的定义和符号}

“概念”, 通常英译为 Concept。而 Idea (想 法）， Thought（思想）, Notion（概念）, Impression (印象) 是 Concept 的同义词。虽然 Concept 和 Notion 均中译为 “概念” , 但 Concept 是
指对事物整体或者本质的感观, 印象与看法; 而 Notion 是指对事物的总体印象, 非常朦胧, 尤其指一 些你认为不对的事情的概念。对旧事物涉及的概念, 明确的数学概念等, 通常用 Concept; 对新事物涉及 的概念, 一些朦朦胧胧才形成的概念, 则多用 Notion。

传统逻辑认为, 词项的含义即概念。例如 “商 品” 一词, 其含义 “为交换而生产的劳动产品” 即为 概念。概念是事物特有属性的反映。

概念史研究者们认为, 概念是思想的出口。通过 对历史中 “主导概念” 的形成、演变、运用及社会文 化影响的分析, 可揭示历史变迁的特征。

不言而喻, “概念” 是头脑思维活动中的重要成 分, 而且是接触外部世界后产生。人类共识性的 “概 念”，可以通过语言进行交流; 一些非共识性的 “概 念”, 内中有部分共识性成分, 也能进行交流; 完全 个性化的 “概念” , 常常在个人思维活动中发挥重要 作用。“财产” 是一个共识性较高的概念, “风险” 是一个非共识性概念, 而 “某人对我很好” 则是一个 完全个性化的概念。一些概念很简单（例如 “水” ) ; 一些概念则非常复杂（例如 “文化”）。

显然, 思维活动中的 “概念” , 远比数理逻辑中 的 “概念” 复杂, 并非都可以用内涵和外延来表达, 也不是用 “对合性” ${ }^{[8]}$ 对内涵和外延加以约束就能解 决问题。

从智能数学研究的需要出发, 本文给出 “概念” 的定义如下:

定义 2. 人类在认识过程中, 把所感知的事物的共同 本质特点抽象出来, 加以概括, 从感性认识上升到理 性认识, 所形成的一种表达, 称为概念, 记为 $\psi$ 。

符号 是一个甲骨文，对应现代汉语中的 “牛” 字。在智能数学中, 为方便书写, 在不引起混淆的情 况下, 本文用斜体英文大写字母 $A, B, \ldots$, 标记不同 的概念。

必须强调的是, 定义 2 不是一个普适性定义, 仅 仅是为研究智能数学而提出。

\section{2. “知识” 的定义和符号}

“知识”, 常英译为 Knowledge。Understanding ( 理解) , Comprehension (理解) , Grasp ( 把 握), Grip (掌握) 被认为是 Knowledge 的同义词。 Understanding 侧重于知道或感知, 而 Comprehension 意味着抓住或捕捉到。

在哲学中, 对知识的研究被称为认识论。两千多 年前, 希腊哲学家柏拉图将知识定义为 “合理的真实 
信仰”。三百年多前, 英国哲学家培根提出 “知识就 是力量” 这个口号。今天, 关于知识的定义, 仍是哲 学家们持续辩论的问题。柏拉图的知识三标准, 被认 为条件不充分, 追加条件之一, 是要求知识 “追踪真 相” [9]。

在心理学中, 个体通过与环境相互作用后获得的 信息被称之为知识。心理学家们将知识分为陈述性知 识和程序性知识。前者是描述客观事物的特点及关系 的知识, 并分三种不同水平: 符号表征、概念、命 题; 后者是一套关于办事的操作步骤。这类知识主要 用来解决 “做什么” 和 “如何做” 的问题。

知识, 至今并没有一个统一而明确的界定, 但有 一些基本的共识, 简单而言, 知而识之就叫知识。换 言之, 对事物知而识之所形成的认识, 均可称为知 识。例如, “地球绕着太阳转”、“房地产泡沫不可 能永远膨胀下去” 和 “6 度及以上地震区的建筑须设 防” 等, 都是知识。一些知识很简单 (例如 “ $1+1=2$ ”) ; 一些知识则非常复杂 (例如 “相对 论” )。据此, 本文给出 “知识” 的定义如下:

定义 3. 人类通过对某类事物的大量感知, 形成对该 类事物具有指导意义的结构化的认识, 称为知识, 记为情。

“知识” 的符号, 由甲骨文 $\psi$ (牛) 和 (马) 并列于直线上方构成, 意为 “牛马都能在地里干活” 这一知识。在智能数学中, 为方便书写, 在不引起混 淆的情况下, 本文用表达函数的字母 $f, g, \ldots$, 标记不 同的知识。

因为知识的类型非常多, 表示知识的方法也就很 多。仅在知识工程中就多达九种: 介谓词逻辑法、产 生式法、框架法、脚本法、过程法、语义网法、 Petri 网法、面向对象法、状态空间表示法等等。定 义 3 的记法, 是为用智能数学描述思维活动时, 让人 们对哪些成分是知识, 哪些不是, 能一目了然。

\section{3. “意识” 的定义和符号}

正如上一节所述, 人类对 “意识” 的奥秘知之甚

少, 很难给其下一个普适性定义。

然而, 数学的严密性又要求界定研究对象, 从智 能数学研究的需要出发, 本文给出意识的定义如下:

定义 4. 人脑中由本能、经验和知识形成的, 据以对 自身行为进行控制的依据, 称为意识。

例如, 人们对风险现象的理解并形成风险管理的 依据, 称为风险意识。
意识有本能意识和后天意识之分。

任何不依赖于经验的行为, 都称为本能 （Instinct）。刚刚在海滩上孵化的海龟具有自动移向 海洋的本能。本能行为也称为先天行为。人脑中由本 能形成的, 据以对自身行为进行控制的依据, 称为本 能意识 (Instinctive Consciousness), 也称为先天意 识。本文以一支电灯泡符号 $\mathrm{x}$ 来记本能意识。

本能行为不一定受大脑控制。本能并不等于本能 意识。只有觉醒后, 才有意识。例如, 婴儿饿了, 会 哭闹, 说明生存本能意识觉醒了。如果婴儿出生时就 有某种脑功能障碍, 其求生的本能仍在, 但 “饿了就 哭闹” 的本能意识可能觉醒不了。

人脑中由经验和知识形成的, 据以对自身行为进 行控制的依据, 称为后天意识 (Acquired Consciousness), 记为被。

没吃早餐的考生在考场饿了, 经验性的意识使之 暂时不进食; 面对滋味鲜美的河豚, 毒性知识形成的 意识, 迫使食客动筷前三思。

图 1 是 “后天意识” 符号放大图。该符号是一支 电灯泡里土壤中有一棵小树, 意为在个体特质制约 下，接触外部世界生长出来的意识。

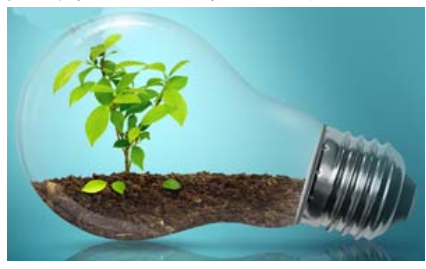

图 1. 后天意识, 无如灯泡里土壤中一棵生长的小 树。灯泡代表意识受个体特质的制约。土壤和阳 光代表意识生长的条件。

在智能数学中, 为便于区别本能意识和后天意 识, 本文用外形封闭的大写希腊字母 $\Delta, \Theta$ 标记本能 意识, 用外形开放的大写希腊字母 $\Omega, \Psi$ 标记后天意 识。

仅就个体的经验意识而言, 其内容不断地变化, 非常复杂。为了概括来自外界或内部无意识中的某些 信息、情感、欲望以连续运动的方式进、出意识的过 程, 人们创造出意识流(Stream of Consciousness) 这 个词。

人脑 “做梦” 被认为是意识流的流动过程。根据 意识流内容的不同, 意识流被分为信息意识流、情绪 意识流、欲望意识流, 而根据意识流的来源和形式的 不同, 又把意识流分为内吸流、内闯流、外吸流、外 闯流。

本文用感作为意识流的符号, 并用@, \$, !,... 标 记不同的意识流。其中, @是电了邮件地址的标志 
性符号, 可代表通讯交流中的意识流; \$是美元符 号, 可代表人们在经济活动中的意识流; ! 是感叹 号，可代表人们做决断时的意识流。

值得特别注意的是, 物质世界中的流体, 显然不 是意识流的原像。流体力学模型, 无法描述意识流。

\section{4. 形成意识流的结构图}

在现实生活与科学探索中, 人们通过观察和思考 来对社会乃至自然界末知世界进行认知, 意识流在进 行思考的思维活动中发挥着举足轻重作用。不经过大 脑思考的本能意识和后天生成的意识, 都是意识流的 组成部分。意识流中的成分与观察得到的信息, 左右 着思维活动的结果。

意识流是一种很有效的小说创作方式。小说以人 物意识活动为结构中心来展示人物持续流动的感觉和 思想, 而且通常借助自由联想来完成叙事内容的转 换。

在目前的人工智能研究中, 人们能训练了一个复 杂的数字神经网络, 实现在不熟悉和黑暗的环境中导 航, 已经是很高的 “智能” 水平了，但与意识流支撑 下的创造性思维相比, 完全不在一个层次。

通过对意识流的分析, 本文认为, 意识流是按图 2 的结构图形成的。

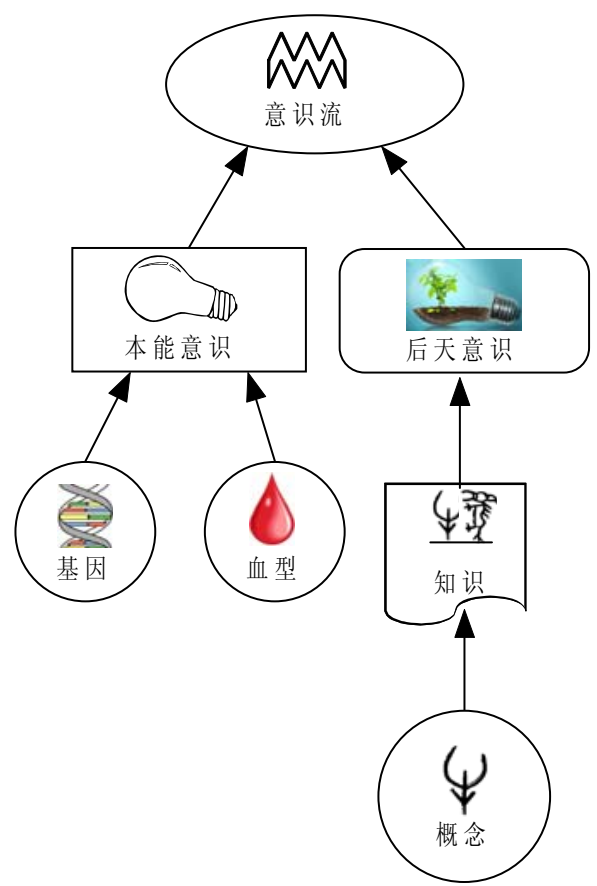

图 2. 意识流中流动着本能 (先天) 意识和后天意 识。基因和血型决定着先天意识。包含经验的知识, 生长 出后的意识。概念支撑着经验和知识的形成。
在意识流中, 本能意识是生命的看护者, 后天意 识中有创造性思维动因。

知识是后天意识生长的土壤, 人脑觉醒的意识是 种子。没有种子, 土壤中长不出后天意识。存储了天 量知识的机器, 没有意识的种子, 出现不了后天意 识, 不能进行创造性思维。

\section{4. 智能数学框架}

以描述意识为基础, 以人类思维活动为研究对象 的智能数学, 涉及从概念生成到思想交流全过程的数 学描述问题。

用因素空间理论可描述概念的生成; 用信息扩散 理论等可描述概念上形成的知识; 知识沉淀则知识上 升为后天意识; 目标驱动使意识上升为意识流动; 思 想在智联网上的交流, 能集小智慧为大智慧。因素空 间、信息扩散和智联网, 构成了一个简单的智能数学 框架。

\section{1. 用因素空间理论描述概念}

因素空间 (Factor Space) 的最初思想, 是汪培 庄在解释随机性的根源及概率规律的数学实质时产生 的。1982 年在文献[10]中给出了一个传统数学意义上 的定义。

“因素” 是一个泛泛的概念, 既指构成事物本质 的成分, 也指决定事物成败的原因或条件, 又称因 子。生物学中的 “基因” , 最初就被称为 “因素” 。 在科学试验中, 影响试验指标的要素或原因, 称为因 素。

在数学上, 因素被定义成映射: 设 $U$ 是所要讨 论的一类事物的集合, 叫作论域。一个定义在论域 $U$ 上的映射 $F: U \rightarrow X$ 叫作 $U$ 上的一个因素, 其中 $X$ 是 映射 $F$ 所映照出来的事物性状的集合。例如, 因素 “身高” 可表示为特定的人群 $U$ 上的一个映射 $H: U$ $\rightarrow[10,250](\mathrm{cm})$, 它把张三映射成实数 $185 \mathrm{~cm}$, 把李 四映射成 $175 \mathrm{~cm}$ 等等。“身高” 这一因素 $H$, 是我 们过去所熟悉的变量, 变量只是一种特殊的因素, 因 素就是变量的推广。

由一些因素组成的集合, 称为因素空间。所有笛 卡尔空间, 例如力学的运动空间、控制论的状态空 间、模式识别的特征空间等, 都是因素空间。它们都 可作为因素空间的特例, 因素空间是笛卡尔空间的推 广。

无论因素是否为变量, 无论因素间是何关系, 任 何事物都可根据它的因素, 将其映射成为因素空间中 
的一个点, 因素空间为一般事物的描述提供了普适性 的数学框架。

因素空间理论认为, 概念产生于比较, 比较发现 异同, 而 “因素” 是概念的划分器 ${ }^{[11]}$, 因此, 可用因 素空间理论描述概念。

"概念", 是人脑区分万物差异的一种表达。婴儿 生存的本能把母亲从万物中区分出来, 在脑海中形成 “母亲” 这一概念。认知的需求, 概念由粗变细。古 人用甲骨文 $\psi$ 将牛从动物中区分出来。中这一表达, 是一个概念。为了进一步认知牛, 细分又出现了耕 牛、奶牛和野牛等等概念。

因素空间理论采用 “内涵” (Intension) 的方式 来描述概念。此处的 “内涵” , 是在因素空间中定义 的 ${ }^{[12]}$ 。

对因素集合 $\boldsymbol{F}=\left\{F_{1}, F_{2}, \cdots, F_{n}\right\}$, 其因素空间记 为:

$$
\Lambda=(\boldsymbol{U}, \boldsymbol{X}(\boldsymbol{F}))
$$

式中, $\Lambda$ 是希腊字母 $\lambda$ 的大写, $\boldsymbol{U}$ 是各因素 $F_{1}, F_{2}, \ldots$, $F_{n}$ 论域 $U_{1}, U_{2}, \ldots, U_{n}$ 的笛卡尔积 $U_{1} \times U_{2} \times \ldots \times U_{n}, \quad \boldsymbol{X}$ 是 $F_{1}, F_{2}, \cdots, F_{n}$ 所映照出来集合 $X_{1}, X_{2}, \cdots, X_{n}$ 的笛卡尔积 $X_{1} \times X_{2} \times \ldots \times X_{n}$ 。

定义 5. 给定因素空间 $\Lambda=(\boldsymbol{U}, \boldsymbol{X}(\boldsymbol{F}))$ ，对任意 $\boldsymbol{a}=\left(a_{1}, a_{2}, \cdots, a_{n}\right) \in \boldsymbol{X}$ ，记其在 $\boldsymbol{U}$ 上的原相为

$$
[\boldsymbol{a}]=\boldsymbol{F}^{-1}(\boldsymbol{a})=\{\boldsymbol{u} \in \boldsymbol{U} \mid \boldsymbol{F}(\boldsymbol{u})=\boldsymbol{a}\}
$$

若 $[\boldsymbol{a}] \neq \phi$, 则称 $[\boldsymbol{a}]$ 是一个内涵颗粒。

$\boldsymbol{a}$ 只是 $\boldsymbol{X}$ 中的一个点, 涉及 $n$ 个因素。 $\boldsymbol{X}$ 中的每 一个点, 在 $\boldsymbol{U}$ 上都有原相, 非空 (即 $[\boldsymbol{a}] \neq \phi$ ), 则称 内涵颗粒。 $\boldsymbol{X}$ 中全体具有内涵颗粒的点组成的集合记 为 $R$, 即:

$$
R=\boldsymbol{F}(\boldsymbol{U})=\left\{\boldsymbol{a}=\left(a_{1}, a_{2}, \cdots, a_{n}\right) \in \boldsymbol{X},[\boldsymbol{a}] \neq \phi\right\}
$$

称 $R$ 为因素 $F_{1}, F_{2}, \cdots, F_{n}$ 之间的背景关系，也叫做因素 $\boldsymbol{F}$ 的背景集。

因素空间理论用 $\boldsymbol{a},[\boldsymbol{a}]$ 和 $R$ 来定义 “概念” :

定义 6. 给定因素空间 $\Lambda=(\boldsymbol{U}, \boldsymbol{X}(\boldsymbol{F}))$, 设 $R$ 是因素 $\boldsymbol{F}=\left\{F_{1}, F_{2}, \cdots, F_{n}\right\}$ 的背景关系, 则对任意 $\boldsymbol{a} \in R$, 称 $a=(\boldsymbol{a},[\boldsymbol{a}])$ 为原子概念, $\boldsymbol{a}$ 和 $[\boldsymbol{a}]$ 分别叫做概念 $a$ 的原 子内涵和原子外延。

此定义告诉我们, 只要因素背景关系知道了, 它 的每一个颗粒就决定一个原子概念。由原子概念用
“且” 字连接起来，可以生成其他的所有概念。

\section{2. 用信息扩散理论描述知识的形成}

信息扩散（Information Diffusion）的最初思想, 是本文作者在研究震害面积和震级间的关系考虑如何 使少量地震记录发挥更大作用时产生的。严格的定 义, 在 1997 年的文献[13]中给出。信息扩散的基本思 想是: 通过有限的观测去认识无限的世界时, 观测到 的样本点是大量尚未观测到样本点的代表。于是, 可 用扩散函数, 将一个没有几何大小的样本点, 转换为 一个涵盖 “周围” 的模糊集, 据此总结出较好的 “知 识”来。

人脑在学习过程中, 由已知联想到未知而总结出 规律, 是从概念上升为知识的必由之路。信息扩散方 法，是进行联想的方法之一。

当然, 人脑的联想, 不仅在知识的形成过程中出 现, 意识流中的联想, 更是创造性思维必不可少。但 是, 目前具有联想功能的信息扩散方法, 只提供了在 概念框架上, 用较少的观测样本点, 估计一个函数来 表达知识的手段。

设 $A, B$ 是可用因素空间描述的两个概念, 例 如, $A$ 是 “地震” 概念, $B$ 是 “损失” 概念。 $A$ 和 $B$ 的关系, 是一种可用函数来表达的知识。用信息扩散 理论描述这种知识的形成, 首先是观测 $A$ 出现时 $B$ 的 情况, 每次观测得到的一个记录, 称为一个样本点。 $A$ 以 $x$ 的面目出现时, 如果 $B$ 的情况是 $y$, 样本点记 为 $(x, y)$, 它是 $A, B$ 所在因素空间中的一个点。此处 的 $x$ 和 $y$ 不一定是实数, 由因素的性质而定。例如, “地震” 不一定是用实数定义的震级, 可以是分成等 级的烈度。

假定对 $A, B$ 的相关现象观测了 $n$ 次, 得样本:

$$
W=\left\{\left(x_{1}, y_{1}\right),\left(x_{2}, y_{2}\right), \ldots,\left(x_{n}, y_{n}\right)\right\}
$$

当 $x$ 和 $y$ 均是实数时, 在概念 $A$ 和概念 $B$ 上形成 的知识 $A B$, 就是从 $W$ 学习到的 $x$ 和 $y$ 的函数关系 $y=f(x)$ 。有大量的传统数学方法可以估计 $f$, 例如, 统计回归和人工神经元网络。但要较好地估计出 $f$, 传统数学方法对样本 $W$ 的要求较高, 例如, 统计 回归中的 $W$ 必须是大样本, 否则统计结果无意义; 人工神经元网络中的 $W$ 不能存在矛盾样本点, 否则 网络学习不收玫。信息扩散方法, 能较好地解决相关 问题, 用 $W$ 较好地估计出 $f$ 。

定义 7. 设概念 $A$ 和 $B$ 的值域分别是 $U$ 和 $V$, 观测样 本 $W$ 中的点 $w=(x, y)$ 联想到 $U \times V$ 中 “周围” 点 $o=(u$, $v$ )的方式 


$$
\mu(w, o)=\exp \left[-\frac{(x-u)^{2}}{2 h_{x}^{2}}-\frac{(y-v)^{2}}{2 h_{y}^{2}}\right]
$$

称函数 $\mu$ 将 $w$ 携带的信息对 “周围” 点 $o$ 进行了正态 扩散。 $\mu$ 称为正态信息扩散函数。

概念的值域, 是指满足概念的所有对象组成的集 合。例如, “牛” 这一概念的值域, 由天下所有的牛 组成。定义 7 中我们用与因素空间中论域相同的字母 记值域, 是因为它们本质上并无区别。

式(5)中扩散系统 $h_{x}$ 和 $h_{y}$, 可根据观测样本 $W$, 用式 (6) 计算。

$$
h_{g}= \begin{cases}0.8146\left(b_{g}-a_{g}\right), & n=5 ; \\ 0.5690\left(b_{g}-a_{g}\right), & n=6 ; \\ 0.4560\left(b_{g}-a_{g}\right), & n=7 ; \\ 0.3860\left(b_{g}-a_{g}\right), & n=8 ; \\ 0.3362\left(b_{g}-a_{g}\right), & n=9 ; \\ 0.2986\left(b_{g}-a_{g}\right), & n=10 ; \\ 2.6851\left(b_{g}-a_{g}\right) /(n-1), & n \geq 11 .\end{cases}
$$

式中, $g \in\{x, y\}, b_{g}=\max _{1 \leq i \leq n}\left\{g_{i}\right\}, a_{g}=\min _{1 \leq i \leq n}\left\{g_{i}\right\}$.

$$
\text { 令 }
$$

$$
q_{o}=\sum_{w \in W} \mu(w, o)
$$

称

$$
Q=\left(q_{o}\right)_{o \in U \times V}
$$

为样本 $W$ 经正态信息扩散得到的关于概念 $A$ 和 $B$ 之间关 系的信息矩阵。

根据 $Q$ 的性质, 采用适当的方法, 由 $Q$ 可以生成 $U \times V$ 上的一个因果型模糊关系, 它是概念 $A$ 和概念 $B$ 上 的知识 $A B$ 。

\section{3. 用智联网集小智慧为大智慧}

智能数学最终是否能帮助人们研制出有意识, 达 到人类智能水平的机器人, 目前还很难定论。在人们 尚无数学手段描述知识沉淀上升为后天意识, 更无法 描述目标驱动使本能意识和后天意识上升为意识流动 的情况下, 人机交互系统, 或许是提高人工智能水平 最有效的途径。自动机能发挥优势的部分, 由机器去 做; 自动机做不了的部分, 由人类来完成。智联网的 出现, 为人机融合提高智能水平, 推动智能数学的发 展, 提供了一个实验平台。

智联网 (Internet of Intelligences) 的最初思想,
是本文作者为提供风险分析在线服务提出的 ${ }^{[14]}$ 。在 2014年的文献[15]中规范化了智联网的定义。为避免 与本文上述已用符号的重复, 智联网定义改写为:

定义 8. 设 $\mathcal{A}$ 是一个智能体集合， $\mathcal{N}$ 是 $\mathcal{A}$ 使用的一 个网络, $\mathcal{M}$ 是处理 $\mathcal{A}$ 所提供信息的模型, 三元体 $<\mathcal{A}, \mathcal{N}, \mathcal{M}$ 称为一个智联网, 记为 $\Phi$ 。

这里的智能体, 主要是指具有观察、演绎、推理 和解决问题能力的个人; 网络主要是指互联网。

智联网的基本思想是: 在数据库和嵌入模型的支 持下, 智能体通过提供信息和参与处理柔性信息, 集 个体小智慧为群体大智, 提供在线服务。

针对具体问题, 能拥有信息和经验参与智联网 解答问题的人并不多。大多数情况下, 智联网是在小 样本的条件下进行工作。由于信息扩散技术能优化处 理小样本，显著提高分析精度，信息扩散模型在智联 网中发挥了核心技术的作用 ${ }^{[16]}$ 。一个简单智联网的拓 扑结构见图 3。智联网的工作原理见图 4。

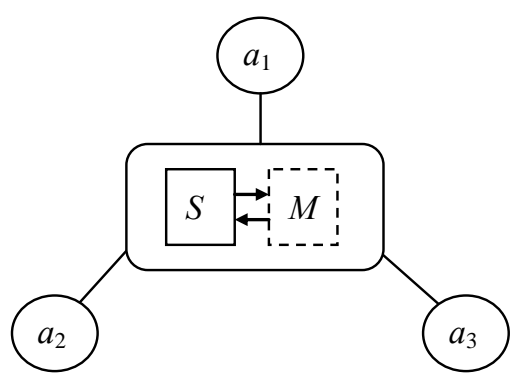

图 3. 一个简单智联网的拓扑结构 ${ }^{[15]}$, 由一个网络服 务器 $S$, 一个数学模型 $M$ 和的三个智能体 $a_{1}, a_{2}, a_{3}$ 组成。

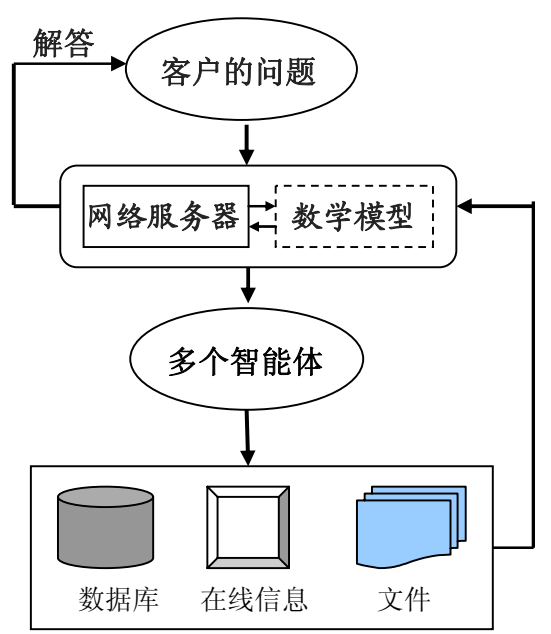

图 4. 智联网工作原理 ${ }^{[15]}$ 。智联网用数学模型处理来 自智能体的在线信息, 在一些背景数据库的支持下, 智联 网为客户解答问题。 
依据图 5 所示的智联网编程原理, 目前, 人们已 经研制出 10 个智联网平台。高考志愿填报智联网服 务平台 ${ }^{[16]}$ 是首个研制成功的智联网演示系统。考生以 购买服务的方式, 通过智联网获得信息和咨询, 并由 数学模型处理后提供决策参考, 有效规避填报失误的 风险。台风灾害风险分析智联网 ${ }^{[17]}$ 中出现了柔性知识 捕获器, 以在温州地区开展台风灾害水产养殖保险的 可行性需求调查为例, 对智联网平台的理念和模型进 行了成功的验证。智联网支撑的 “优秀论文” 评选系 统 ${ }^{[18]}$ 能评出共识性最高的优秀论文, 多次用于中国风 险分析与管理精英杯优秀论文的评选。内涝风险分析 智联网服务平台 ${ }^{[19]}$ 使用属性拼图技术对原始的经验信 息进行优化整合, 再通过 “雨强一水深” 模糊关系模 型, 在线实现基于降雨强度, 估计易涝点积水深度, 进而给出积水风险值。智联网驱动的风险雷达 ${ }^{[20]}$ 通过 社区居民的参与, 进行风险信息搜集、动态风险评 估, 实现了风险事件的动态追踪。该项发明已由北京 崇安智联科技服务有限责任公司申请了名为 “一种社 区安全风险雷达智联网服务系统” 的发明专利。海洋 环境风险管理智联网平台 ${ }^{[21]}$ 通过去中心化信息收集的 技术, 完成了围填海造地项目对天津自然灾害抵御能 力的影响评价。地震宏观异常的智联网服务平台 ${ }^{[22]}$ 将 众多一线地震工作者对地震宏观异常群强度的目视判 断, 以模糊隶属函数的曲线方式输入, 在智联网中建 立以模糊关系矩阵表达的共识性宏观异常群测度空 间, 有望为震前宏观异常辅助地震预报提供帮助。风 险时效性评价的智联网服务平台 ${ }^{[23]}$ 是一个因素藤智联 网, 被用于对北京延庆区果树冰䨌灾害概率风险的时 效性进行评价。风险沟通智联网服务平台 ${ }^{[24]}$ 提供了洪 水灾害风险沟通的便捷渠道, 并尝试用于宁波市洪水 灾害的风险管理。四川省三台县永和堰灌区综合风险 评估智联网信息系统 ${ }^{[25]}$, 用开源的WebGIS 代码库

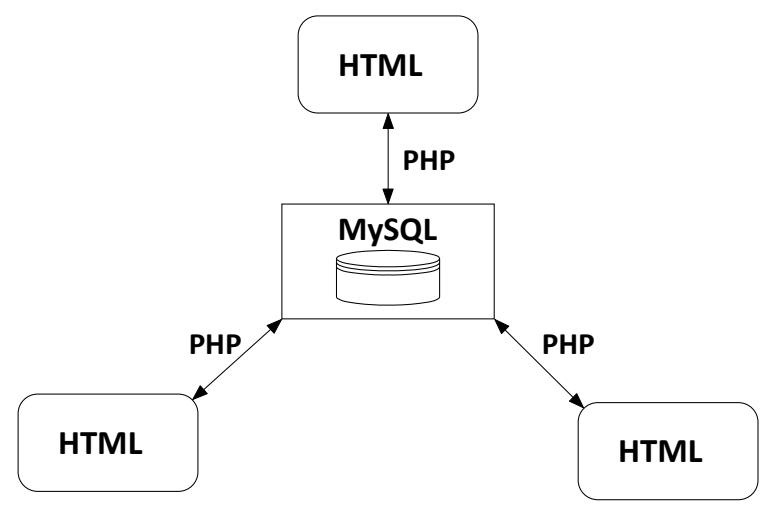

图 5. 智联网编程原理示意图 ${ }^{[2]}$ 。智联网平台由三个部 分构成: 终端界面、服务器上的数据库和连接界面和数据 库并能运行数学模型的网络程序。它们分别由HTML语 言、MySQL数据库和PHP语言来实现。
Leaflet调用自制图层, 实现了地理信息系统与智联 网的融合, 首次不依赖于第三方Web 地图API就能为 智联网平台提供网络地图服务。

\section{4. 简单智能数学框架图}

由于在描述概念方面的优势, 因素空间理论有望 成为智能数学的基础。例如, 针对专门概念, 用因素 空间理论帮助设计专用的智联网, 其上发布的问题更 明确, 智能体的响应更有效。由于在处理小样本方面 的优势, 信息扩散理论有望成为智能数学中的一个学 习算法, 模拟人脑由已知联想到未知, 形成知识。由 于在集小智慧为大智慧方面的优势, 智联网有望成为 智能数学的实验平台, 推动智能数学的形成和发展。 据此, 本文用图6给出一个简单的智能数学框架。
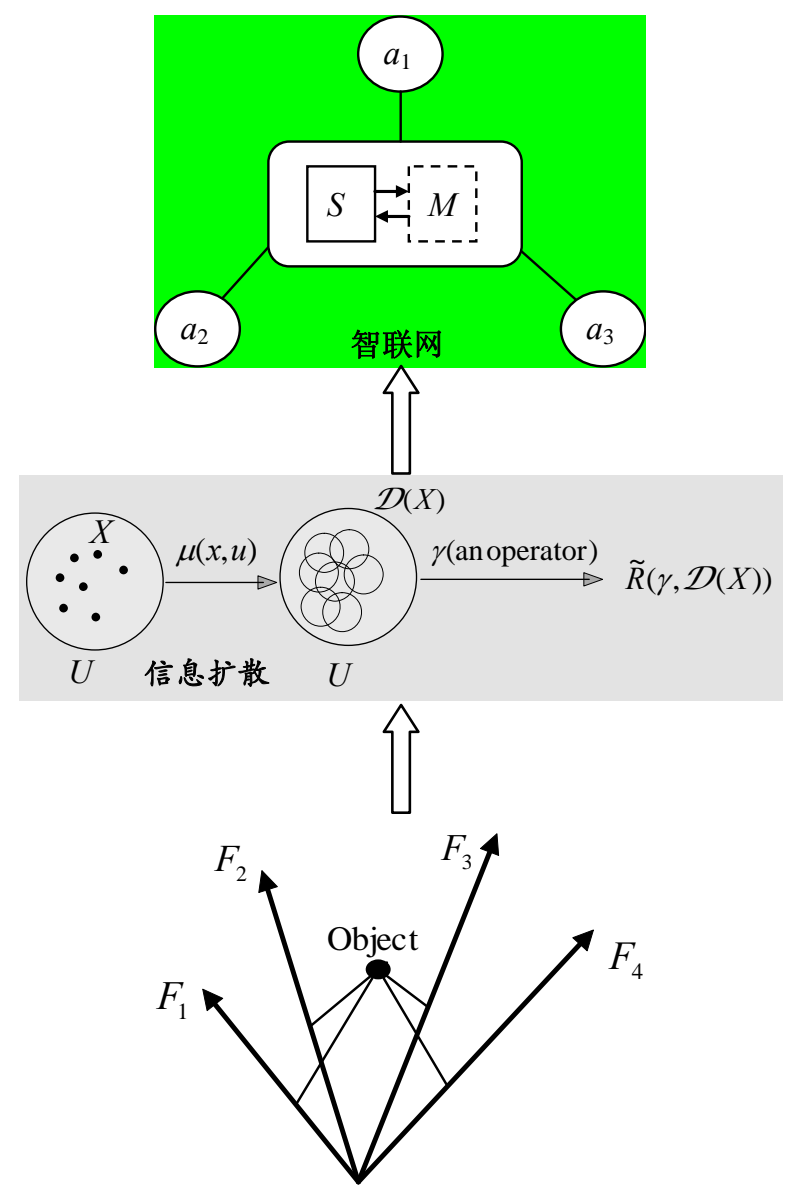

因素空间

图 6. 一个简单的智能数学框架。任何事物都是某个因素 空间中的一个点, 因素空间可描述概念; 信息扩散以联想 方式学习而获得知识 $\widetilde{R}$; 智联网提供人机交互平台, 集小 智慧为大智慧。 


\section{5. 用智能数学描述地震风险感知}

地震风险感知, 是典型的 “不确定环境下对多个 对象及综合后果的研判” 行为, 研判的水准, 体现了 智能水平的高低。其中, 涉及两类对象: 一类是与地 震有关的对象, 它们是地震发生的时间、空间和强 度; 另一类是与人类社会有关的对象, 它们是地震造 成的死亡、伤害和损失。个体观测并感悟这两类对 象, 对综合后果进行研判, 是个体对地震风险的感 知。利益相关群体对研究区地震风险感知的基本共 识, 就是人们对该地区的地震风险感知。最主要的利 益相关者, 是当地的民众, 其次是当地的官员和企业 家, 最后是生活在该地区以外, 但对这一地区及周边 的地震风险有研究的专家。

本文用智能数学来描述这种地震风险感知。

\section{1. 用因素空间理论描述地震风险概念}

地震风险, 对不同的人有不同的理解。媒体人理 解的地震风险, 较具弹性, 侧重于感知和表述; 公众 的文化背景不同, 对地震风险有不同的解释; 科技人 员理解的地震风险, 主要是基于概率思维和统计学计 算的数字形式。如果不是在约定的概念下进行观测和 感悟, 一群人感知出来的地震风险就没有意义。

本文用一个二阶因素藤来描述地震风险概念。因 素藤是通过子因素来定义的 ${ }^{26]}$ 。给定因素 $F, G$, 如果 $F$ 的状态决定了 $G$ 的状态, 即 $G$ 的状态空间是 $F$ 状态 空间的子空间, 此时称 $G$ 是 $F$ 的子因素。例如, 地震 时、空、强是地震因素的 3 个子因素; 死、伤、损是 震害因素的 3 个子因素。由具有层次关系联系的多个 因素空间联结体, 称为因素藤(Factor Vine)。只有两 层结构因素藤称为二阶因素藤。

设 $F_{1}$ 和 $F_{2}$ 分别是地震和震害因素, $G_{1}, G_{2}$ 和 $G_{3}$ 分别是地震时、空、强因素, $G_{4}, G_{5}$ 和 $G_{6}$ 分别是 死、伤、损因素, 则对地震风险的一次认识, 就是图 7 所示两层结构中一个点 Object。此两层结构图, 是 有两个分枝的因素藤。

本文建议用图 8 是来表达此因素藤, 使之形象 化。这种表达, 也称为多层结构的藤化表达法。

在欧几里得空间中, 数轴之间必须正交。在因素 空间中, 因素之间不必正交, 而且还允许因素是定性 的性状, 这就使得因素藤结构非常灵活性, 可以描述 非常复杂的概念。

\section{2. 用信息扩散方法学习地震灾害知识}

破坏性地震记录, 是形成地震灾害知识的依据。 不同地区的地震特征和社会形态不一样, 形成的知识

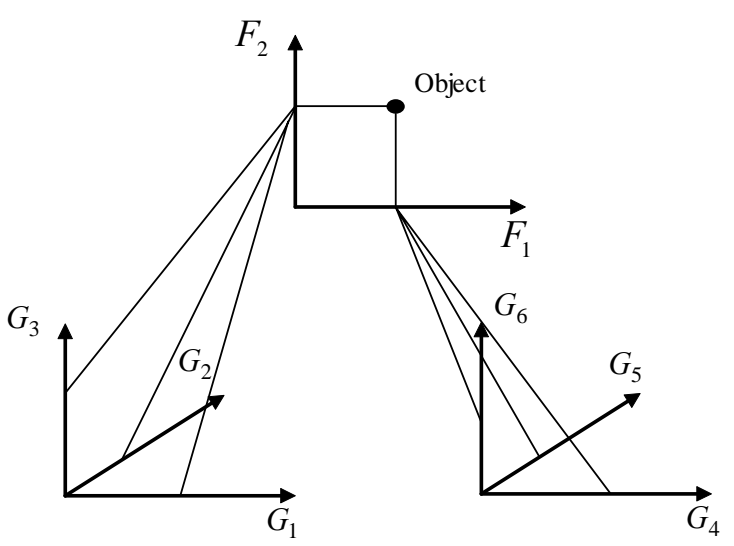

图 7. 用于描述地震风险概念的两层因素空间结构。

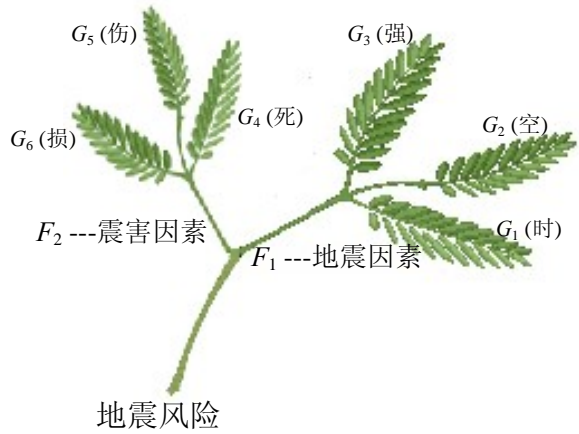

图 8. 地震风险概念的因素藤。 $G$ 类因素是 $F$ 类因素的子因 素。不同人感知出来的地震风险, 均须在此因素藤的影响 空间之中, 方可进行沟通和综合。

也就不一样。一个地区的地震灾害知识, 主要由两部 分组成, 一部分是关于地震发生的频度; 另一部分是 地震强度与灾害程度的关系。前者用概率分布表达, 后者用易损性曲线表达。

不失一般性, 设某地区在过去的 $T$ 年 (通常是几 十年) 内共发生了 $n$ 次破坏性地震, 第 $i$ 次地震的里 氏震级为 $x_{i}$, 造成灾害的程度记为向量 $\boldsymbol{y}_{i}$, 由死、 伤、损三个分量组成。这 $n$ 次破坏性地震记录形成一 个样本:

$$
W=\left\{\left(x_{1}, \boldsymbol{y}_{1}\right),\left(x_{2}, \boldsymbol{y}_{2}\right), \ldots,\left(x_{n}, \boldsymbol{y}_{n}\right)\right\}
$$

当我们将一个地区视为内部无差异性的整体时, 描述地震发生地点的子因素 “空” , 不再发挥作用; 当我们将灾害程度看作是只受地震强度的影响, 而与 在 $T$ 年内发生于何时无关时, 子因素 “时” 也不再发 生作用。 $\boldsymbol{y}$ 中的死、伤、损, 分别由该地区死亡和失 踪总人数, 致残人数, 用消费者物价指数校正后的经 济损失数据 (货币化) 计之。 $x$ 和 $\boldsymbol{y}$ 的值域分别记为 $U$ 和 $\boldsymbol{V}$ : 


$$
\begin{aligned}
& U=\left\{u_{1}, u_{2}, \ldots, u_{J}\right\} \\
& \boldsymbol{V}=\left\{\boldsymbol{v}_{1}, \boldsymbol{v}_{2}, \ldots, \boldsymbol{v}_{K}\right\} \\
& \text { 令 } \\
& q_{i j}=\exp \left[-\frac{\left(x_{i}-u_{j}\right)^{2}}{2 h_{x}^{2}}\right], i=1,2, \cdots, n ; j=1,2, \cdots, J \\
& S_{j}=\sum_{i=1}^{n} q_{i j}
\end{aligned}
$$

则, 用信息扩散方法, 通过学习式 (9) 中的样本 $W$, 我们得到该地区破坏性地震发生概率分布的一个估 计:

$$
p\left(u_{j}\right)=\frac{S_{j}}{n}, \quad j=1,2, \cdots, J
$$

在用信息扩散方法学习易损性曲线时, 样本点 中的灾害向量 $\boldsymbol{y}_{i}$ 和值域中点 $\boldsymbol{v}_{k}$ 的距离用范数 $\|\cdot\|$ 记之, 即

$$
d\left(\boldsymbol{y}_{i}, \boldsymbol{v}_{k}\right)=\left\|\boldsymbol{y}_{i}-\boldsymbol{v}_{k}\right\|
$$

因为灾害向量 $\boldsymbol{y}_{i}$ 的三个分量死、伤、损均是实数, 此 范数为欧氏距离。设 $\boldsymbol{y}_{i}=\left(y_{1 i}, y_{2 i}, y_{3 i}\right), \boldsymbol{v}_{k}=\left(v_{1 k}, v_{2 k}, v_{3 k}\right)$, 则

$$
\left\|\boldsymbol{y}_{i}-\boldsymbol{v}_{k}\right\|=\sqrt{\left(y_{1 i}-v_{1 k}\right)^{2}+\left(y_{2 i}-v_{2 k}\right)^{2}+\left(y_{3 i}-v_{3 k}\right)^{2}}
$$

于是, 样本点 $w_{i}=\left(x_{i}, \boldsymbol{y}_{i}\right)$ 扩散到值域点 $o_{j k}=\left(u_{j}, \boldsymbol{v}_{\boldsymbol{k}}\right)$ 的信 息量是:

$$
\begin{array}{r}
\mu\left(w_{i}, o_{j k}\right)=\exp \left[-\frac{\left(x_{i}-u_{j}\right)^{2}}{2 h_{x}^{2}}-\frac{\left\|\left(\boldsymbol{y}_{i}-\boldsymbol{v}_{k}\right)\right\|^{2}}{2 h_{y}^{2}}\right] \\
=\exp \left[-\frac{\left(x_{i}-u_{j}\right)^{2}}{2 h_{x}^{2}}-\frac{\left(y_{1 i}-v_{1 k}\right)^{2}}{2 h_{y_{1}}^{2}}\right. \\
\left.-\frac{\left(y_{2 i}-v_{2 k}\right)^{2}}{2 h_{y_{2}}^{2}}-\frac{\left(y_{2 i}-v_{2 k}\right)^{2}}{2 h_{y_{3}}^{2}}\right]
\end{array}
$$

令

$$
Q_{j k}=\sum_{i=1}^{n} \mu\left(w_{i}, o_{j k}\right)
$$

则, 用信息扩散方法, 通过学习式(9)中的样本 $W$, 我们得到该地区关于易损性的一个信息矩阵:

$$
Q=\begin{gathered}
u_{1} \\
u_{2} \\
\vdots \\
u_{J}
\end{gathered}\left[\begin{array}{cccc}
Q_{11} & \boldsymbol{v}_{2} & \cdots & \boldsymbol{v}_{K} \\
Q_{21} & Q_{22} & \cdots & Q_{1 K} \\
\vdots & \vdots & \vdots & \vdots \\
Q_{J 1} & Q_{J 2} & \cdots & Q_{J K}
\end{array}\right]
$$

我们对 $Q$ 进行列向归一化处理, 形成 $W$ 所在 地区地震强度 $(u)$ 和震害程度 $(\boldsymbol{v})$ 因果关系的 知识, 即模糊关系 $f$ :

$$
f=\begin{gathered}
v_{1} \\
u_{1} \\
u_{2} \\
\vdots \\
u_{J}
\end{gathered}\left[\begin{array}{cccc}
r_{11} & r_{12} & \cdots & r_{1 K} \\
r_{21} & r_{22} & \cdots & r_{2 K} \\
\vdots & \vdots & \vdots & \vdots \\
r_{J 1} & r_{J 2} & \cdots & r_{J K}
\end{array}\right]
$$

式中，

$$
\left\{\begin{array}{l}
r_{j k}=Q_{j k} / H_{k} \\
H_{k}=\max _{1 \leq j \leq J}\left\{Q_{j k}\right\}
\end{array}\right.
$$

即, 用信息矩阵中各列的最大值遍除该列中的所 有元素，生成模糊关系。于是，根据模糊近似推 理 ${ }^{[27]}$, 当地区内发生里氏震级 $u_{j}$ 时, 由总结出来的 知识 $f$, 可以推导出震害程度是一个模糊集, 如式 (22) 所示。

$$
\tilde{\boldsymbol{V}}=\frac{r_{j 1}}{\boldsymbol{v}_{1}}+\frac{r_{j 2}}{\boldsymbol{v}_{2}}+\cdots+\frac{r_{j k}}{\boldsymbol{v}_{k}}+\cdots+\frac{r_{j K}}{\boldsymbol{v}_{K}}
$$

上式为模糊集的扎德表述法, 其 “分母” 是式 (11) 中值域 $\boldsymbol{V}$ 的元素, “分子” 是向量元素 $\boldsymbol{v}$ 属于模糊集 $\tilde{\boldsymbol{V}}$ 的程度。加号 “+” 是用来进行罗列, 当隶属度为 0 时, 那一项可以不写入。采用式 (23) 的重心法, 可以估计出死亡和失踪人数 $v_{1}$ 、致残人数 $v_{2}$ 和经济损 失 $v_{3}$ 。

$$
v_{l}^{(j)}=\left(\sum_{k=1}^{K} r_{j k} v_{l k}\right) /\left(\sum_{k=1}^{K} r_{j k}\right), \quad l=1,2,3 .
$$

\section{3. 在智联网中描述地震风险感知}

我们用因素空间理论描述了地震风险是图 8 因素 藤表达的概念, 信息扩散方法学习式(9)的历史地震记 录 $W$, 形成了式(20)的因果关系知识。

当我们以震害程度的期望向量 $\boldsymbol{v}$ 作为地震风险的 内涵时, 用式(14)的概率分布和式(23)的易损性值, 可分别估计出死亡和失踪人数期望值、致残人数期望 值和经济损失期望值。

如果在未来的 $T$ 年内, 该地区的地震活动规律在 统计意义上与过去 $T$ 年内的情况完全一样, 并且经济 社会情况也没有任何的变化, 那么, 依据 $W$ 估计出 来的, 式(24)期望值, 就是在未来的 $T$ 年内, 该地区 的地震风险。 


$$
R_{l}=\sum_{j=1}^{J} p\left(u_{j}\right) v_{l}^{(j)}, \quad l=1,2,3 .
$$

然而, 事实并非如此。第一个问题是, 不仅地区 内有地震活动规律会有变化, 周边地区的强烈地震也 会影响所研究地区, 未来 $T$ 年内的破坏性地震不可能 仍然是式(14) 的概率分布。况且, 如果人们对地震风 险的关注是较短时期内, 例如 5 年内的地震风险, 则 依据 $W$ 估计出的概率分布, 并不能直接使用。第二 个问题是, 随着人民生活水平的提高或遭遇变故, 经 济社会情况肯定会发生明显变化。

将 $W$ 所提供的地震灾害知识, 以 “统计风险 值” 示人, 展现给在智联网上参与地震风险感知的智 能体, 针对未来 $\tau$ 年内该地区的地震风险, 发挥他们 的历史感知、现实感知和未来感知的意识流动作用, 各人分别给出感知的地震风险值。再考虑参与者关于 风险的保守、中性和侥幸取向, 综合各人给出的风险 值, 得出样本 $W$ 所来自地区未来 $\tau$ 年内地震风险感知 值。

在智联网中描述地震风险感知, 由三个部分组 成:（1）计算统计意义上的风险值;（2）激活个体 的风险感知意识流; (3) 综合群体感知的结果。

\subsection{1. 计算统计意义上的风险值}

由授权的人员, 通过智联网的交互界面, 将式(9) 的历史地震记录 $W$ 放入服务器的数据库中。

智联网依次用公式(6)的程序计算扩散系数, 式 (12)-(14)计算概率分布, 式(17)、(18)和(21) 学习因果 关系, 最后用式(23)和(24)计算出死亡和失踪人数风 险值 $R_{1}$ 、致残人数风险值 $R_{2}$ 和经济损失风险值 $R_{3}$ 。

在智联网学习风险知识的过程中, 用到的值域 $U$ 和 $\boldsymbol{V}$, 是以 $W$ 相应分量观测值两点最小间距为步 长。

由智联网将 $R_{1} 、 R_{2}$ 和 $R_{3}$ 值推送到所研究地区的 地图界面, 并推送其依据 $W$ 到该界面。

从 $W$ 到 $R_{1} 、 R_{2}$ 和 $R_{3}$, 是用智能数学中的因素空 间理论和信息扩散描述了对地震风险的一种感知, 类 似于人脑形成后天意识的一小部分。

在这一过程中, 因素空间理论承担了界定 “地震 风险概念” 的工作, 决定了 $W$ 的成分。例如, $W$ 中不 包含 “旱” 的成分。尽管有专家认为, 震前一至三年 半时间内, 旱区面积大, 则震级大 ${ }^{[28]}$, 但 “旱” 与 “震” 并无直接关系, 旱不能作为地震风险概念的因 素使用。

在约定的地震风险概念基础上, 依据一个地区的 历史地震资料, 形成该地区地震风险统计知识的过程
中, 信息扩散方法以联想的方式, 描述了如何用资料 来感知风险。

因素空间的概念分析和信息扩散的联想学习, 这 两部分智能数学的工作, 都可以在智联网上自动实 现, 其智能水平, 可以用与其它方法的估计误差来标 定。但是, 目前仍须借助利益相关者的参与, 方能更 真实地感知所研究地区的地震风险。这就要求智联网 能激活个体的地震风险感知意识流。

\subsection{2. 激活个体的风险感知意识流}

假定在智联网上参与地震风险感知的所有个体, 都对所研究地区具有地震风险意识, 亦即是说, 这些 个体对该地区的地震风险有所了解，并对地震风险管 理有所思考。这与网络上漫无际地发表评论及千里之 外坐而道论的风险评估, 完全不同。

具有该地区地震风险意识的个体, 沉淀风险知识 上升为后天意识。风险感知的目标驱动, 使他的本能 意识和后天意识上升为意识流, 以完成风险感知的任 务。风险意识流左右思维活动感知风险, 是一个非常 复杂的意识流过程。

虽然目前没有数学手段可以描述感知风险的意识 流, 但人机交互的智联网, 可以通过提供信息和提出 问题, 触发意识流。研究区景物和历史地震在脑海中 的出现, 是意识流的起点; 地震风险概念的提示和统 计风险信息的获得, 提供了意识流的初始方向; 被邀 请充分发挥个体能动性感知地震风险, 为意识流注入 了活力; 面对地震, 与生俱来的生存本能, 日常生活 经验和方方面面的知识, 都在左右感知地震风险的意 识流。每个参与者, 都会形成独一无二的地震风险感 知结果。我们用图 9 来展现从起点到终点的个体意识 流。

\subsection{3. 综合群体感知的结果}

每个人的风险感知, 都是独一无二的, 除非规定 用同一个风险分析模型, 处理同一批数据资料, 得出 同样的结果。但是, 这不是风险感知, 而是机械计 算。

个体的地震风险感知结果, 不仅受经验和知识的 影响, 而且与保守、中性或侥幸取向有极大的关系。 承受过地震灾害的人, 生存本能使之取向保守, 不自 觉地放大风险; 没有承受过地震灾害又不涉及公共资 源的人, 取向较为中性; 对于能得到投资或项目而从 中获利者, 发展本能使之取向侥幸, 不自觉地缩小风 险。 


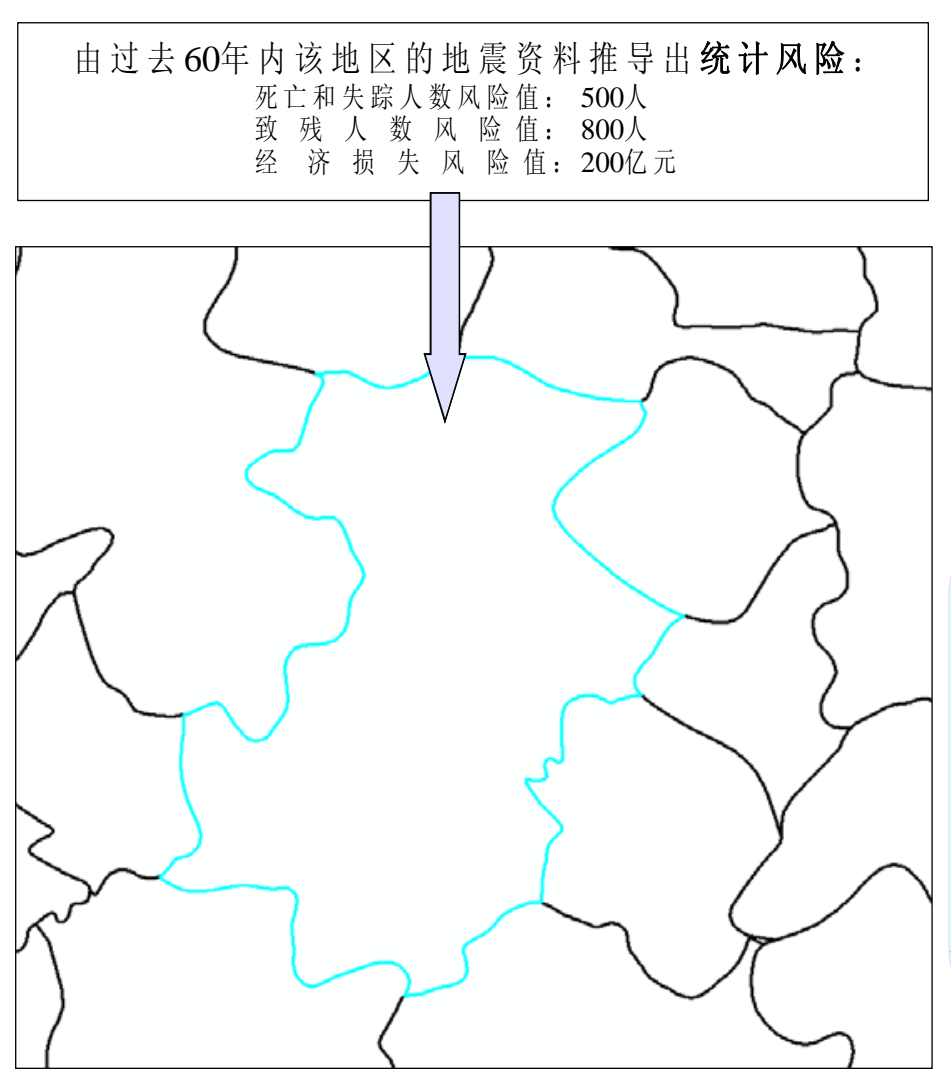

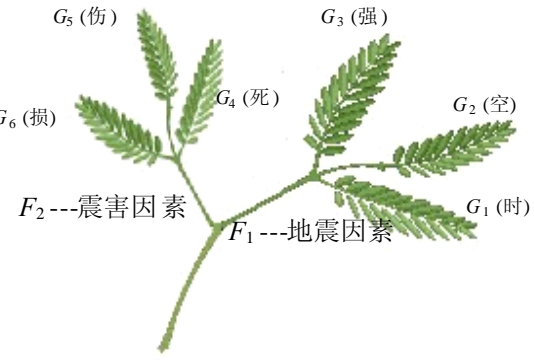

地震风险

\section{尊敬的地震风险感知者:}

请以上图约定的地震风险概念, 参考统计风险值，考虑近年及将来 地震活动性和经济社会变化及周边 可能发生地震的影响, 对该地区未 来10年内风险值三个分量进行估计。 我估计死亡和失踪人数期望值是: 我估计致残人数期望值是: 我估计经济损失期望值是 (亿元):

我通常以保守 $\boldsymbol{V}$ 的心态面对风险问题

图 9. 激活参与者意识流进行地震风险感知的智联网交互界面。研究区及周边地区地图, 是意识流的起点; 统计 风险和因素藤信息, 为意识流提供了初始方向; 各种变化及周边影响进入意识流, 感知的地震风险更具可解释 性; 未来 10 年内风险值的估计, 是意识流左右思维活动产生的结果。

在智联网中综合群体感知的结果，须对保守 (Conservation）、中性(Impartial)和侥幸(Lucky)型的 结果分别处理后再综合。

假定在 $N$ 个结果中, 这三种类型分别有 $N_{C}$, $N_{I}, N_{L}$ 个, 且 $N=N_{C}+N_{I}+N_{L}$ 。

$\forall \eta \in\{C, I, L\}$ ，该类型第 $l$ 个分量的风险值记为 $r_{l}^{(\eta)}$ 。于是 $\eta$ 类型的个体, 他们的地震风险感知结果 形成一个集合:

令

$$
R_{l}^{(\eta)}=\left\{r_{l 1}^{(\eta)}, r_{l 2}^{(\eta)}, \cdots, r_{l N_{\eta}}^{(\eta)}\right\}
$$

$$
\bar{R}_{l}^{(\eta)}=\frac{1}{N_{\eta}} \sum_{i=1}^{N_{\eta}} r_{l i}^{(\eta)}, \quad l=1,2,3 ; \eta \in\{C, I, L\}
$$

则

$$
R_{l}=\frac{\bar{R}_{l}^{(C)}+\bar{R}_{l}^{(I)}+\bar{R}_{l}^{(L)}}{3}, l=1,2,3
$$

为群体感知的综合结果。其中, $l=1,2,3$ 分别代表死亡 和失踪风险, 致残风险和经济损失风险。

为什么说智联网是智能数学的一部分, 我们只要 将其视为一个映射结构, 就能一目了然, 只不过目前 还不能将映射过程中出现的 “意识流”书写出来。

用三种基本元素: 数字、算子和结构, 传统数学 中就能机械地描述物质世界, 但仅用这三种元素, 描 述不了人类意识。除了本文建议增加的 “概念”

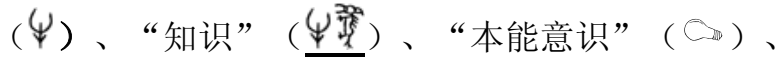
“后天意识”（ 外, 还须有相应的数学算子和数学结构。

式(5)的正态信息扩散, 是一个联想式数学算 子; 图 8 的地震风险概念因素藤, 是一个数学结构; 图 9 背后的智联网, 是一个以研究区地图和统计风险 为原像, 以参与者群体的意识流为映射函数, 以风险 估计值为像的一个映射结构。与传统数学的映射结构 不同，目前还无法描述智联网中的意识流映射函数。 
而且, 同样的原像, 同样的参与群体, 在不同时间 映射出来的像, 不会完全相同。例如, 同样 30 个人 的群体对一个地区的地震风险感知, 今天的风险估计 值与昨天的值就不会完全相同, 因为受开放环境的影 响, 参与者在这两天的情绪, 会有所差异。一个好的 智联网结构, 应该在相距不长时间前后, 结果相差不 大。

\section{6. 结论与讨论}

现代科学意义上的风险分析, 本质上是在进行风 险感知。在相当一段时间内, 地震风险感知, 仍须人 工参与。要减少风险感知中的人工参与, 须有真正的 机器智能出现。目前被高度商业化包装的 “人工智 能”, 只是自动化意义下的智能, 而不是智慧意义下 的智能。

以代数学和微积分为根基的现代数学, 难以将 “人工智能” 推进到智慧型机器智能, 因为其研究对 象的原像均在物质世界中。只有出现能描述意识, 以 人类思维活动为研究对象的智能数学, 才可能出现超 越自动化的人工智能。

数字、算子和结构是传统数学中的基本元素, 远 不能支撑智能数学, 我们最起码须考虑加入 “概念” 等符号作为智能数学的基本元素。“概念” 显然比数 字和数学变量复杂得多。

由因素空间、信息扩散和智联网可以组成一个简 单的智能数学框架。因素空间中的因素藤, 是一个智 能数学结构, 用于描述概念; 信息扩散中的正态信息 扩散模型, 是一个智能数学算子, 用于联想式学习小 样本形成知识; 智联网是一个意识流映射函数, 同时 也是一种须有人工参与的智能数学结构。

用现有的数学来研究智能问题, 类似于用机械鸟 来实现飞行的梦想。用智能数学来研究智能问题, 则 类似于用有一定向上凸起弧度的机翼来实现飞行。思 路不同，效果肯定不同。

风险分析, 尤其是对复杂而动态的风险进行分 析, 是最能考验智能水平的一项工作。于是, 各显神 通的数学模型, 天罗地网的监测系统, 纷纷登场; 于 是, 各种风险曲线, 花花绿绿的风险图, 铺天盖地。 然而, 人们在这一领域取得的成功, 远在自动化的人 工智能之下。原因之一, 是人们缺少智能数学来提高 风险分析的质量。

本文建议的, 由因素空间、信息扩散和智联网组 成的智能数学框架, 虽然简单, 但相比文 [2]仅仅提出 “将第三代数学称为智能数学” , 是一个进步。但愿 人们能走出 “用传统数学的零件, 组装出智能数学 来” 的空想, 脚踏实地面对诸 “地震风险感知” 需要
人类智慧才能解决的问题, 解决一些传统数学方法解 决不了的问题。

\section{参考文献}

[1] 黄崇福.自然灾害风险分析与管理. 北京: 科学出版社, 2012.

[2] Huang C F. Principle of internet of intelligences and development of its core technology. Journal of Risk Analy-sis and Crisis Response, 2017, 7(3): 146-155.

[3] 刘云丰,陈洪,刘忠,史光亚. 智能数学:内容、方法及意义. 自然杂志,1987,(4):255-262.

[4] http:/int-math.com

[5] Anastassiou G A. Intelligent Mathematics: Computational Analysis. Heidelberg: Springer-Verlag, 2011.

[6] 钟义信. 机制主义人工智能理论一一种通用的人工智 能理论. 智能系统学报, 2018, 13(1): 2-18.

[7] 何华灿. 泛逻辑学理论一一机制主义人工智能理论的逻 辑基础. 智能系统学, 2018, 13(1): 19-36.

[8] Wille R. Restructuring lattice theory: An approach based on hierarchies of concepts, in Ordered Sets, I. Rival (Ed.) (Dordrecht: Springer, 1982), pp. 445-470.

[9] Nozick R. Philosophical Explanations. Cambridge, Massachusetts: Harvard University Press, 1981.

[10] 汪培庄,Sugeno M. 因素场与模糊集的背景结构. 模糊 数学, 1982,(2):45-54.

[11] 汪培庄. 因素空间理论一一机制主义人工智能理论的 数学基础. 智能系统学报, 2018, 13(1): 37-54.

[12] Wang P Z, Ouyan H, Zhong Y X, He H C. Cognition math based on factor space. Annals of data science, 2016, 3(3): 281-303.

[13] Huang C F. Principle of information diffusion. Fuzzy Sets and Systems, 1997, 91(1): 69-90.

[14] Huang C F. Internet of intelligences in risk analysis for online services. Journal of Risk Analysis and Crisis Response, 2011, 1(2): 110-117.

[15] Huang C F. Multiple internet of intelligences for risk analysis. Journal of Risk Analysis and Crisis Response, 2014, 4(2): 61 - 71.

[16] 北京崇安智联科技服务有限责任公司. 高考志愿填报 智联网服务平台一联接智慧, 网获知识 (available at: http://www.cazl.cn/ioiserver/index.php?r=site/main), 2013.

[17] 艾福利. 自然灾害风险分析智联网服务平台构建与应 用研究. 北京师范大学博士学位论文,2014.

[18] 北京崇安智联科技服务有限责任公司. 风险学会 “优 秀论文奖” 评选系统(available at: http://www.cazl.cn/ risktech/yxlw/),2014.

[19] 曾凡雷. 属性拼图智联网风险分析方法及其服务平台 的构建与应用研究. 北京师范大学博士学位论文, 2016.

[20] Huang C F, Wu T, Renn O. A risk radar driven by inter- 
net of intelligences serving for emergency management in community. Environmental Research, 2016, 148: 550559.

[21］苏妩. 信息收集去中心化在风险评估中的应用---海洋 环境风险管理智联网平台研究. 北京师范大学硕士学 位论文,2016.

[22] 王蔚丹. 地震宏观异常的综合度量及其在智联网服务 平台上的应用研究. 北京师范大学博士学位论文,2017.

[23] 郭君. 自然灾害概率风险时效性评价的因素空间架构 体系及其在智联网上的实现. 北京师范大学博士学位 论文,2017.

[24] 梁屹. 网络互动模式下洪水灾害风险沟通共识获得的 方法一基于智联网途径. 北京师范大学博士学位论 文,2017.

[25] 孙雁南.与智联网相融合的地理信息系统在风险分析 中的应用一以四川省三台县永和堰灌区的脆弱性评价 为例. 北京师范大学硕士学位论文,2018.

[26] 汪培庄. 因素空间与概念描述. 软件学报, 1992, (1): 30-40.

[27] 黄崇福. 自然灾害风险分析的信息矩阵方法. 自然灾害 学报, 2006, 15(1): 1-10.

[28] 耿庆国. 旱震关系与大地震中期预报. 中国科学(B 辑), 1984, (7): 568-667. 